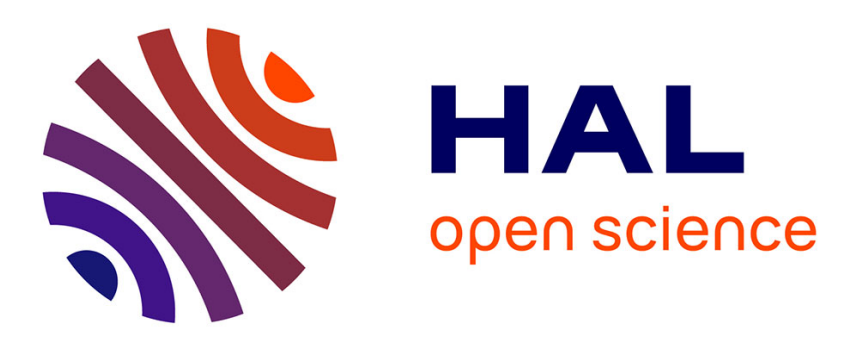

\title{
Rapid stabilization of a reaction-diffusion equation with distributed disturbance
}

Patricio Guzmán, Christophe Prieur

\section{To cite this version:}

Patricio Guzmán, Christophe Prieur. Rapid stabilization of a reaction-diffusion equation with distributed disturbance. CDC 2020 - 59th IEEE Conference on Decision and Control, Dec 2020, Jeju Island (virtual), South Korea. 10.1109/CDC42340.2020.9304185 . hal-03192474

\section{HAL Id: hal-03192474 \\ https://hal.science/hal-03192474}

Submitted on 8 Apr 2021

HAL is a multi-disciplinary open access archive for the deposit and dissemination of scientific research documents, whether they are published or not. The documents may come from teaching and research institutions in France or abroad, or from public or private research centers.
L'archive ouverte pluridisciplinaire HAL, est destinée au dépôt et à la diffusion de documents scientifiques de niveau recherche, publiés ou non, émanant des établissements d'enseignement et de recherche français ou étrangers, des laboratoires publics ou privés. 


\title{
Rapid stabilization of a reaction-diffusion equation with distributed disturbance
}

\author{
Patricio Guzmán and Christophe Prieur
}

\begin{abstract}
In this paper we address the problem of rapid stabilization of a reaction-diffusion equation with distributed disturbance. With the aid of the spectral decomposition of the spatial operator associated to the equation and the sign multivalued operator, which is used to reject the effects of the disturbance, we design a feedback law that exponentially stabilizes, with decay rate as large as desired, the corresponding infinite-dimensional system. The well-posedness of the resulting closed-loop system, which actually is a differential inclusion, is shown with the maximal monotone operator theory.
\end{abstract}

\section{INTRODUCTION}

Reaction-diffusion equations arise naturally in a variety of models from biology, chemistry and physics as can be seen in [27], [25], [2], [6] and the references therein. This kind of model is suitable for many applications because it allows to describe distributed parameter systems, and also, dynamics depending on both the time and another variable of interest, as the space. For such infinite-dimensional systems, dedicated control techniques are available in the literature to analyze the stability or to design stabilizing controllers. Classical tools are Lyapunov control methods [9], [21] and backstepping techniques [18], useful for both linear and nonlinear partial differential equations (see also [8]).

The present work considers the case of an unstable reaction-diffusion equation with disturbances. Both the control and the disturbance operators are bounded, to model a dynamical system with in-domain inputs (one control and one disturbance). For this kind of perturbed distributed parameter systems, some methods for stability analysis and control have been developed, as the input-to-state stability analysis [22] or the direct study of Sturm-Liouville operators [17].

In this paper we address the problem of rapid stabilization of a reaction-diffusion equation with distributed disturbance. Roughly speaking, we solve a stabilization problem of an infinite-dimensional system by ensuring a decay rate as large as desired. Moreover, we also obtain that the stability of the closed-loop system is robust with respect to some unknown distributed disturbances.

This study has been partially supported by ECOS-CONICYT C16E06. The work of the first author is partially supported by FONDECYT 3180363. The work of the second author is partially supported by MIAI @ Grenoble Alpes, ANR-19-P3IA-0003.

P. Guzmán is with Departamento de Matemática, Universidad Técnica Federico Santa María, Valparaíso, Chile.

patricio.guzmanm@usm.cl

C. Prieur is with Université Grenoble Alpes, CNRS, Grenoble INP, GIPSA-lab, Grenoble, France.

christophe.prieurlgipsa-lab.fr
Let us introduce more precisely the control problem under consideration in this paper. Let $\Omega \subset \mathbb{R}^{N}$, with $N$ in $\mathbb{N}$, be an open and bounded set of class $C^{1}$. Its boundary is denoted by $\partial \Omega$. Let $a$ in $(0, \infty)$ be the diffusion coefficient and $c$ in $L^{\infty}(\Omega)$ be the reaction term. Let us consider the reactiondiffusion equation

$$
\left\{\begin{array}{l}
z_{t}-a \triangle z-c z=u+d,(t, x) \in(0, \infty) \times \Omega, \\
z(t, x)=0,(t, x) \in(0, \infty) \times \partial \Omega, \\
z(0, x)=z_{0}(x), x \in \Omega .
\end{array}\right.
$$

The purpose of this paper is to design a controller so that the closed-loop system is exponentially stable in $L^{2}(\Omega)$, with decay rate as large as desired. This is done by means of a feedback law $u=u(t, x)$ that rejects the effects of a disturbance $d=d(t, x)$. Although we assume that the disturbance is unknown, later we make some assumptions on its regularity and its $L^{2}(\Omega)$ norm.

Let us note that (11) is an unstable partial differential equation. Indeed, in the undisturbed case, which is when the disturbance is zero, the term $c^{+}(x)=\max \{c(x), 0\}$, which corresponds to the non-negative part of the reaction term $c \in L^{\infty}(\Omega)$, is the source of instability in $(1)$. Then, in order to exponentially stabilize 11 in $L^{2}(\Omega)$, so that the decay rate is at least $\omega \in(0, \infty)$, we could take the feedback law

$$
u(t, x)=\left(-c^{+}(x)-\omega\right) z(t, x) .
$$

We could also take other feedback laws, such as those constructed from a finite-dimensional truncated system as in [14], [19], [20] and [26], for instance. Regarding the disturbed case, it is uncertain whether we can employ the same feedback laws we would use in the undisturbed case, since in their construction no information of the disturbance is used, and hence, such feedback laws might not be able to handle the effects of the disturbance. This scenario has been observed in [11, Page 193] for the wave equation with distributed disturbance and in [15, Page 96] for the Euler-Bernoulli equation with boundary disturbance, for instance. Accordingly, in (1) we may regard the disturbance as another source of instability.

In order to reject the effects of the disturbance we use the sign multivalued operator defined in [3, Section 4.2.3]. Given a Hilbert space $\mathscr{H}$ we define the sign multivalued operator $\operatorname{sign}_{\mathscr{H}}(\cdot): \mathscr{H} \rightarrow 2^{\mathscr{H}}\left(2^{S}\right.$ denotes the power set of $\left.S\right)$ by 


$$
\operatorname{sign}_{\mathscr{H}}(f)=\left\{\begin{array}{c}
\frac{f}{\|f\|_{\mathscr{H}}} \text { if } f \neq 0, \\
\left\{g \in \mathscr{H} /\|g\|_{\mathscr{H}} \leq 1\right\} \text { if } f=0 .
\end{array}\right.
$$

The sign multivalued operator has shown to be useful to deal with the effects of a disturbance, acting either in the domain or at the boundary, as can be seen in [12], [13], [11], [15], [16] and [24], for instance. In all those works, 33 has been used with $\mathscr{H}=\mathbb{R}$. Nevertheless, due to technical reasons in this paper we use $\sqrt{3}$ with $\mathscr{H}=L^{2}(\Omega)$.

In order to present our main result we need to make the following two assumptions on the disturbance.

(A1) $d \in L^{1}\left(0, \infty ; L^{2}(\Omega)\right)$.

(A2) There exists $D \in[0, \infty)$ such that $\|d(t, \cdot)\|_{L^{2}(\Omega)} \leq D$ for $t \in[0, \infty)$.

Our main result is the following one.

Theorem 1: Let us assume (A1) and (A2). Let $z_{0}$ in $L^{2}(\Omega)$ be the initial condition. Let $\omega$ in $(0, \infty)$ be the desired decay rate. Then, there exists a feedback operator $\mathscr{F}: L^{2}(\Omega) \rightarrow$ $L^{2}(\Omega)$ such that $(1)$ with the feedback law

$$
u(t, x)=\mathscr{F}(z(t, x))-D \operatorname{sign}_{L^{2}(\Omega)}(z(t, x))
$$

is exponentially stable in $L^{2}(\Omega)$, with decay rate $\omega$. Being more explicit, (1) with the feedback law (4) has a unique weak solution $z$ in $C\left([0, \infty) ; L^{2}(\Omega)\right)$, and it satisfies

$$
\|z(t, \cdot)\|_{L^{2}(\Omega)} \leq e^{-\omega t}\left\|z_{0}\right\|_{L^{2}(\Omega)}, t \in[0, \infty)
$$

Remark 1: Assumption (A1) is required for the wellposedness part of Theorem 1 and assumption (A2) is needed for the construction of the feedback law (4).

Remark 2: The feedback operator $\mathscr{F}: L^{2}(\Omega) \rightarrow L^{2}(\Omega)$ not only exists, we actually provide its precise expression, which is the one given by (20).

In Theorem 1 the feedback law (4) is composed by two parts. The feedback operator is involved in the first part and its role is to achieve the desired decay rate, while in the second part, the sign multivalued operator allows to reject the effects of the disturbance. Let us note that the resulting closed-loop system, obtained by plugging the feedback law (4) into (1), is a differential inclusion due to the presence of the sign multivalued operator. We show its well-posedness by applying the maximal monotone operator theory, which may be consulted in [4], [3] or [28], for instance.
All the literature concerned with the stabilization of disturbed models cited in this paper are of one-dimensional nature. Up to our knowledge, this is the first paper addressing the stabilization of a $\mathrm{N}$-dimensional disturbed model.

The rest of this paper is organized as follows. The proof of Theorem 1 is carried out in Sections III and III Being precise, the feedback law (4) is designed in Section $\mathrm{II}$ and the well-posedness of the resulting closed-loop system is shown in Section III. Then, in Section IV we provide a numerical simulation. Finally, in Section V we give some concluding remarks.

Notation. The partial derivatives are taken in the sense of distributions. The Sobolev space $H^{1}(\Omega)$ is formed by the $y$ in $L^{2}(\Omega)$ such that $\nabla y$ in $L^{2}(\Omega)^{N}$. The Sobolev space $H_{0}^{1}(\Omega)$ is the closure of $C_{0}^{\infty}(\Omega)$ in $H^{1}(\Omega)$. The theory of Sobolev spaces may be consulted in [1], [10, Chapter 5] or [5, Chapter 9], for instance. The space $L^{1}\left(0, \infty ; L^{2}(\Omega)\right)$ are the integrable $y(t, \cdot):(0, \infty) \rightarrow L^{2}(\Omega)$ such that $\|y\|_{L^{1}\left(0, \infty ; L^{2}(\Omega)\right)}=\int_{0}^{\infty}\|y(t, \cdot)\|_{L^{2}(\Omega)} d t<\infty$. The space $C\left([0, \infty) ; L^{2}(\Omega)\right)$ are the continuous $y(t, \cdot):[0, \infty) \rightarrow L^{2}(\Omega)$ such that $\|y\|_{C\left([0, \infty) ; L^{2}(\Omega)\right)}=\max _{t \in[0, \infty)}\|y(t, \cdot)\|_{L^{2}(\Omega)}<\infty$. The space $W^{1,1}\left(0, \infty ; L^{2}(\Omega)\right)$ are the $y$ in $L^{1}\left(0, \infty ; L^{2}(\Omega)\right)$ such that $y_{t}$ in $L^{1}\left(0, \infty ; L^{2}(\Omega)\right)$. Further details of these three vectorvalued functional spaces may be found in [28, Chapter III] or [10, Section 5.9.2], for instance.

\section{FEEDBACK DESIGN}

Let us assume that $z=z(t, x)$ is a regular enough solution of (1). The required regularity for the following formal computations will be justified in Section III. From (1) we get

$$
\begin{gathered}
\frac{1}{2} \frac{d}{d t}\left(\int_{\Omega}|z|^{2} d x\right)+\int_{\Omega}(-a \triangle z-c z) z d x \\
=\int_{\Omega}(u+d) z d x .
\end{gathered}
$$

With the purpose to appreciate the idea behind the feedback design, in (6) we decompose the feedback law as $u=u_{1}+u_{2}$, where $u_{1}$ will be constructed to achieve the desired decay rate and $u_{2}$ will be constructed to reject the effects of the disturbance. Being precise:

- We are going to construct $u_{1}$ such that

$$
\omega \int_{\Omega}|z|^{2} d x \leq \int_{\Omega}\left(-a \triangle z-c z-u_{1}\right) z d x
$$

- We are going to construct $u_{2}$ such that

$$
\int_{\Omega}\left(u_{2}+d\right) z d x \leq 0 .
$$


Let us note that if we are able to construct the feedback law $u=u_{1}+u_{2}$ so that (7) and (8) are satisfied, then we can obtain (5) from (6). Indeed, combining (6)-(8) it follows

$$
\frac{d}{d t}\left(\int_{\Omega}|z|^{2} d x\right)+2 \omega \int_{\Omega}|z|^{2} d x \leq 0,
$$

from which the exponential stability in $L^{2}(\Omega)$ of $(1)$, with decay rate $\omega \in(0, \infty)$, is obtained.

\section{A. Design of $u_{1}$}

We identify $L^{2}(\Omega)$ with its dual space. We would like to view (1) in its operator form. To that end, first let us recall that $\Omega \subset \mathbb{R}^{N}$ is an open and bounded set of class $C^{1}$, and hence, by [1, Theorem 5.37] we have

$$
H_{0}^{1}(\Omega)=\left\{\phi \in H^{1}(\Omega) / \phi=0 \text { on } \partial \Omega\right\} .
$$

Then, let us introduce the operator

$$
\left\{\begin{array}{l}
\mathscr{A}: D(\mathscr{A}) \subset L^{2}(\Omega) \rightarrow L^{2}(\Omega), \\
D(\mathscr{A})=\left\{\phi \in H_{0}^{1}(\Omega) / \triangle \phi \in L^{2}(\Omega)\right\}, \\
\mathscr{A} \phi=a \triangle \phi+c \phi,
\end{array}\right.
$$

which is linear in $\phi$. Since $H^{1}(\Omega) \subset L^{2}(\Omega)$ with compact injection thanks to [5, Theorem 9.16], we can adapt the arguments used in the proof of [5, Theorem 8.22] (see also [5, Theorem 9.31] or [23, Appendix B]) to prove that $\mathscr{A}$ is a self-adjoint operator with compact resolvent. Then, by [5, Theorem 6.11] we can take a Hilbert basis $\left\{\phi_{m}\right\}_{m \in \mathbb{N}} \subset$ $D(\mathscr{A})$ of $L^{2}(\Omega)$ composed by the eigenfunctions of $\mathscr{A}$. Furthermore, the corresponding sequence of eigenvalues $\left\{\lambda_{m}\right\}_{m \in \mathbb{N}} \subset \mathbb{R}$ satisfies

$$
\left\{\begin{array}{l}
\lambda_{m} \geq \lambda_{m+1}>-\infty \text { for each } m \in \mathbb{N} \\
\lambda_{m} \rightarrow-\infty \text { as } m \rightarrow \infty
\end{array}\right.
$$

Let us note that we could take $u_{1}$ as the right-hand side of (2). However, we would be acting on all the eigenvalues, which is not necessary. We proceed to construct $u_{1}$ so that it will only influence the non-negative and slow enough eigenvalues. To that end, let $n \in \mathbb{N}$ be such that

$$
0<\omega \leq-\lambda_{m} \text { if } m \in\{n, n+1, \ldots\} .
$$

By (11) we infer that we can always find such a $n$ in $\mathbb{N}$. Let us consider

$$
z(t, x)=\sum_{m=1}^{\infty} z_{m}(t) \phi_{m}(x) .
$$

Let us choose the gains $G_{m}(t)$, with $m$ in $\{1, \ldots, n-1\}$, of the feedback law

$$
u_{1}(t, x)=\sum_{m=1}^{n-1} G_{m}(t) \phi_{m}(x)
$$

so that (7) is satisfied. Taking into account (10), (13), (14) and the orthonormality of the eigenfunctions it follows

$$
\begin{aligned}
& \int_{\Omega}\left(-a \triangle z-c z-u_{1}\right) z d x \\
& =-\int_{\Omega}\left(\mathscr{A} z+u_{1}\right) z d x \\
& =-\sum_{m=1}^{n-1}\left[\lambda_{m} z_{m}(t)+G_{m}(t)\right] z_{m}(t)-\sum_{m=n}^{\infty} \lambda_{m} z_{m}(t)^{2} .
\end{aligned}
$$

In view of (12), we see in (15) that it suffices to choose the gains $G_{m}(t)$ so that $\lambda_{m} z_{m}(t)+G_{m}(t)=-\omega z_{m}(t)$. Therefore, we obtain

$$
\begin{gathered}
\int_{\Omega}\left(-a \triangle z-c z-u_{1}\right) z d x \\
\geq \sum_{m=1}^{n-1} \omega z_{m}(t)-\sum_{m=n}^{\infty} \lambda_{m} z_{m}(t)^{2} \geq \omega \sum_{m=1}^{\infty} z_{m}(t)^{2},
\end{gathered}
$$

from which we arrive at (7) with the feedback law

$$
u_{1}(t, x)=\sum_{m=1}^{n-1}\left(-\lambda_{m}-\omega\right) z_{m}(t) \phi_{m}(x)
$$

Remark 3: By (11) and (12) we either have $\lambda_{m} \geq 0$ or $0<-\lambda_{m}<\omega$ when $m \in\{1, \ldots, n-1\}$. Accordingly, from (2) together with 16 we can appreciate the influence of $c^{+}$ onto the finite number of non-negative eigenvalues. Actually, if $c^{+}=0$, then we would only have $0<-\lambda_{m}<\omega$ when $m \in\{1, \ldots, n-1\}$, which corresponds to the slow enough eigenvalues.

\section{B. Design of $u_{2}$}

We proceed to construct $u_{2}$ so that $(8)$ is satisfied. To that end, we are going to employ $\sqrt{3}$ with $\mathscr{H}=L^{2}(\Omega)$ and the property

$$
\int_{\Omega} \theta f d x=\|f\|_{L^{2}(\Omega)}, \forall f \in L^{2}(\Omega), \forall \theta \in \operatorname{sign}_{L^{2}(\Omega)}(f) .
$$

Let us recall that the disturbance is unknown, and hence, we cannot choose $u_{2}(t, x)=-d(t, x)$. Thanks to CauchySchwarz inequality we have

$$
\begin{aligned}
& \int_{\Omega}\left(u_{2}+d\right) z d x \\
& \leq \int_{\Omega} u_{2} z d x+\|d(t, \cdot)\|_{L^{2}(\Omega)}\|z(t, \cdot)\|_{L^{2}(\Omega)}
\end{aligned}
$$

Since $\|d(t, \cdot)\|_{L^{2}(\Omega)} \leq D$ by Assumption (A2), from 17) together with (18) we see that it suffices to choose the feedback law

$$
u_{2}(t, x)=-D \operatorname{sign}_{L^{2}(\Omega)}(z(t, x))
$$

to arrive at $(8)$. 


\section{Feedback law}

We proceed to get the feedback law (4). To that end, we first need to construct the feedback operator. Given $f$ in $L^{2}(\Omega)$ let us consider

$$
f(x)=\sum_{m=1}^{\infty} f_{m} \phi_{m}(x) .
$$

With (16) in mind, let us define the feedback operator $\mathscr{F}: L^{2}(\Omega) \rightarrow L^{2}(\Omega)$ by

$$
\mathscr{F}(f)=\sum_{m=1}^{n-1}\left(-\lambda_{m}-\omega\right) f_{m} \phi_{m}(x) .
$$

Then, by (16), 20) and (13) we can write

$$
u_{1}(t, x)=\mathscr{F}(z(t, x)) .
$$

Finally, since $u=u_{1}+u_{2}$, by 211 and $(19)$ we get the feedback law (4), whose explicit form is given by

$$
\begin{aligned}
u(t, x) & =\sum_{m=1}^{n-1}\left(-\lambda_{m}-\omega\right) z_{m}(t) \phi_{m}(x) \\
& -D \operatorname{sign}_{L^{2}(\Omega)}(z(t, x)) .
\end{aligned}
$$

\section{WELL-POSEDNESS}

Let us write the resulting closed-loop system. To that end, let us introduce the multivalued operator

$$
\left\{\begin{array}{l}
\mathscr{B}: D(\mathscr{B}) \subset L^{2}(\Omega) \rightarrow 2^{L^{2}(\Omega)}, \\
D(\mathscr{B})=D(\mathscr{A}), \\
\mathscr{B} \phi=-\mathscr{A} \phi-\mathscr{F}(\phi)+D \operatorname{sign}_{L^{2}(\Omega)}(\phi) .
\end{array}\right.
$$

Then, in virtue of (1), (10), the feedback law (4) and (23) it follows that the resulting closed-loop system is the differential inclusion

$$
\left\{\begin{array}{l}
z^{\prime}(t)+\mathscr{B} z(t) \ni d(t), t>0, \\
z(0)=z_{0} .
\end{array}\right.
$$

In order to prove its well-posedness we are going to apply the maximal monotone operator theory. In that direction, we have the following result.

\section{Proposition 1: $\mathscr{B}$ is a maximal monotone operator.}

Proof. The operator $-\mathscr{A}-\mathscr{F}$ is linear and self-adjoint. Moreover, by (10), (21) and (7) it follows that $-\mathscr{A}-\mathscr{F}$ is a monotone operator. Therefore, by [7, Corollary 2.4.8] we have that $-\mathscr{A}-\mathscr{F}$ is a maximal monotone operator (or equivalently $\mathscr{A}+\mathscr{F}$ is a m-dissipative operator). In our context the notion of maximal monotone operator coincides with the one of $m$-accretive operator. Then, we conclude the result thanks to [3, Chapter 4, Proposition 2.4] applied to the case $X=L^{2}(\Omega)$, where $L^{2}(\Omega)$ is identified with its dual space. There we have considered that the duality mapping $J: X \rightarrow X^{*}$, where $X^{*}$ is the dual space of $X$, is the identity operator in $X$ (see [3, Page 4]).
To complete the proof of Theorem 1 we proceed to show that (1) with the feedback law (4) has a unique weak solution $z \in C\left([0, \infty) ; L^{2}(\Omega)\right)$ and it satisfies $(5$ ).

For the moment let us assume that $d \in W^{1,1}\left(0, \infty ; L^{2}(\Omega)\right)$ and $z_{0} \in D(\mathscr{B})$. Then, [28, Chapter IV, Theorem 4.1] gives the existence of a unique $z \in W^{1,1}\left(0, \infty ; L^{2}(\Omega)\right)$ such that:

$$
\begin{aligned}
& z(0)=z_{0}, \\
& z^{\prime}(t)+\mathscr{B} z(t) \ni d(t) \text { for almost every } t>0, \\
& z(t) \in D(\mathscr{B}) \text { for every } t \geq 0 .
\end{aligned}
$$

Therefore, [28, Chapter III, Proposition 1.2] applies and all the formal computations done in Section [II make sense, implying that (5) is satisfied.

Given $(d, \widehat{d}) \in W^{1,1}\left(0, \infty ; L^{2}(\Omega)\right)^{2}$ and $\left(z_{0}, \widehat{z}_{0}\right) \in D(\mathscr{B})^{2}$, let us take the corresponding unique solution $(z, \widehat{z}) \in$ $W^{1,1}\left(0, \infty ; L^{2}(\Omega)\right)^{2}$. Then, by [28, Chapter IV, (4.12)] we have

$$
\begin{aligned}
& \|z(t, \cdot)-\widehat{z}(t, \cdot)\|_{L^{2}(\Omega)} \leq\left\|z_{0}-\widehat{z}_{0}\right\|_{L^{2}(\Omega)} \\
& +\int_{0}^{t}\|d(s, \cdot)-\widehat{d}(s, \cdot)\|_{L^{2}(\Omega)} d s, t \geq 0 .
\end{aligned}
$$

Taking into account the density of $W^{1,1}\left(0, \infty ; L^{2}(\Omega)\right)$ in $L^{1}\left(0, \infty ; L^{2}(\Omega)\right)$ and of $D(\mathscr{B})$ in $L^{2}(\Omega)$, we may use 25 to define, as in [28, Page 183] for instance, the notion of weak solution of [24]. Therefore, in virtue of [28, Chapter IV, Theorem 4.1A] we have that 24 has a unique weak solution $z \in C\left([0, \infty) ; L^{2}(\Omega)\right)$ and it satisfies 5 provided that $d \in L^{1}\left(0, \infty ; L^{2}(\Omega)\right)$ and $z_{0} \in L^{2}(\Omega)$. This ends the proof of Theorem 1 .

\section{NUMERICAL SIMULATIONS}

To illustrate the obtained stability result, let us consider the reaction-diffusion equation (1) with $N=1$. As far as the operator is concerned without neither control nor disturbance, which is the one given by (10), its eigenvalues when $c \in \mathbb{R}$ are $\lambda_{n}=c-a n^{2} \pi^{2} / L^{2}$ and the corresponding eigenfunctions are $\phi_{n}(x)=\sqrt{2 / L} \sin (n \pi x / L), n$ in $\mathbb{N}$ and $x$ in $[0, L]$. We consider the following data for the space domain $L=2 \pi$, the diffusion coefficient $a=0.5$, the reaction term $c=0.5$, the initial condition $z_{0}(x)=-x(2 L / 3-x)(L-x)$, for all $x$ in $[0, L]$, and the maximal perturbation radius equal to $D=1.25$.

Note that, with these parameter values, the first eigenvalue of the open-loop operator is positive. Moreover, we may check that the scalar product between the initial condition and the first eigenvector is non zero, and thus, without disturbance and without any control the solution to (1) diverges. This open-loop unstability is checked on numerical simulations of Figure 1. 


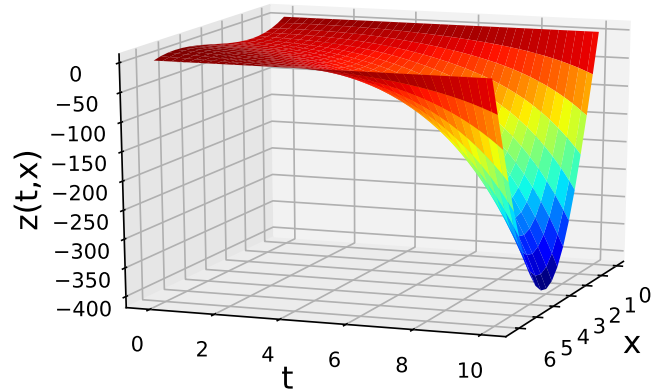

Fig. 1. Time evolution of $z(t, x)$ for the open-loop system without disturbance

The set of coefficient and initial condition is the same as in [19]. In this configuration, we have two unstable modes $\lambda_{1}=0.375$ and $\lambda_{2}=0$. For the perturbation, we set $d(x, t)=$ $D \sin (t)(2 \pi x / L)$. We use a forward Euler methoo 1 with 20 space points. The time-evolution of the closed-loop system is depicted in Figure 2 with the control given in Figure 3. where both parts of the feedback law could be seen, namely $u_{1}$ for small time instant, and $u_{2}$ for larger time instant. As expected from Theorem 1 the system state converges to zero.

\section{CONCLUSION}

In this paper an (unstable open-loop) reaction-diffusion equation has been considered in presence of disturbance and control inputs. A control strategy has been introduced to solve the rapid stabilization problem. In closed-loop with the designed controlled, the solutions to the distributed parameter systems are exponentially stabilized in $L^{2}(\Omega)$, with decay rate as large as desired. The obtained $N$ dimensional reaction-diffusion equation (1) rejects the effects of an unknown distributed disturbance satisfying the assumptions (A1) and (A2). Such task for the feedback law (4) was possible thanks to the sign multivalued operator (3) with $\mathscr{H}=L^{2}(\Omega)$.

This work lets some other control problems open. It could be interesting to consider other (linear or nonlinear) partial differential equations. The obtained performance should also be balanced with respect to the disturbance rejection. This trade-off will be considered in a future work.

\section{REFERENCES}

[1] R. Adams and J. Fournier. Sobolev Spaces. Pure and Applied Mathematics. Academic Press, 2003.

[2] F. Argomedo Bribiesca, E. Witrant, C. Prieur, S. Brémond, R. Nouailletas, and J.-F. Artaud. Lyapunov-based distributed control of the safety-factor profile in a tokamak plasma. Nuclear Fusion, 53(3):033005, 2013.

${ }^{1}$ The simulation code using Python 3 could be reached here https:// github.com/prieurch/SimulationCodes.git

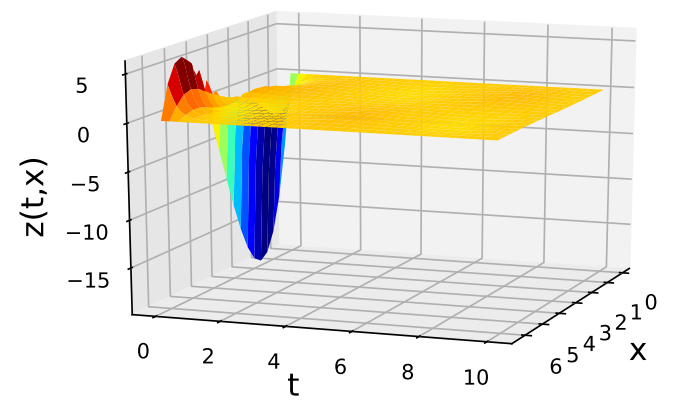

Fig. 2. Time evolution of $z(t, x)$ for the closed-loop system
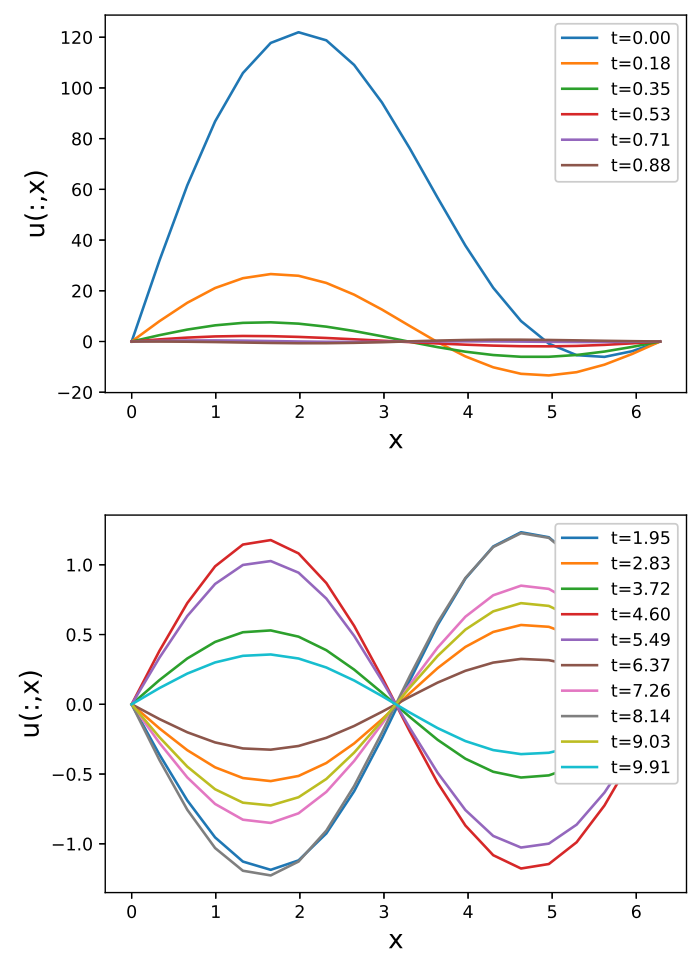

Fig. 3. Feedback law $u$ at different time instant. Top: for time smaller than 1 , down: for time larger than 1

[3] V. Barbu. Analysis and Control of Nonlinear Infinite Dimensional Systems. Mathematics in Science and Engineering. Academic Press, 1993.

[4] H. Brezis. Opératerus maximaux monotones et semi-groupes de contractions dans les espaces de Hilbert. Mathematics Studies. NorthHolland, 1973.

[5] H. Brezis. Functional Analysis, Sobolev Spaces and Partial Differential Equations. Universitext. Springer, 2010.

[6] F. Bribiesca Argomedo, E. Witrant, and C. Prieur. Safety Factor Profile Control in a Tokamak. Springer, 2014.

[7] T. Cazenave and A. Haraux. An Introduction to Semilinear Evolution Equations. Oxford Lecture Series in Mathematics and Its Applications. Oxford University Press, 1998.

[8] J.-M. Coron. Control and Nonlinearity, volume 136 of Mathematical Surveys and Monographs. American Mathematical Society, Providence, RI, 2007. 
[9] R. Curtain and H. Zwart. An introduction to infinite-dimensional linear systems theory. Springer-Verlag, 1995.

[10] L. Evans. Partial Differential Equations. Graduate Series in Mathematics. American Mathematical Society, 2010.

[11] Q.-H. Fu and G.-Q. Xu. Exponential stabilization of 1-d wave equation with distributed disturbance. WSEAS Transactions on Mathematics, 14:192-201, 2015.

[12] B.-Z. Guo and W. Kang. The Lyapunov approach to boundary stabilization of an anti-stable one-dimensional wave equation with boundary disturbance. International Journal of Robust and Nonlinear Control, 24(1):54-69, 2014.

[13] B.-Z. Guo and W. Kang. Lyapunov approach to the boundary stabilisation of a beam equation with boundary disturbance. International Journal of Control, 87(5):925-939, 2014.

[14] P. Guzmán, S. Marx, and E. Cerpa. Stabilization of the linear Kuramoto-Sivashinsky equation with a delayed boundary control. IFAC PapersOnLine, 52(2):70-75, 2019.

[15] F.-F. Jin and B.-Z. Guo. Lyapunov approach to output feedback stabilization for the Euler-Bernoulli beam equation with boundary input disturbance. Automatica, 52:95-102, 2015.

[16] W. Kang and B.-Z. Guo. Arbitrary decay for boundary stabilization of Schrödinger equation subject to unknown disturbance by Lyapunov approach. IFAC Journal of Systems and Control, 7:Article 100033, 2019.

[17] I. Karafyllis and M. Krstic. Input-to-State Stability for PDEs. Communications and Control Engineering. Springer, 2019.

[18] M. Krstic and A. Smyshlyaev. Boundary Control of PDEs: A Course on Backstepping Designs. SIAM, Philadelphia, PA, USA, 2008.

[19] H. Lhachemi, C. Prieur, and R. Shorten. An LMI condition for the robustness of constant-delay linear predictor feedback with respect to uncertain time-varying input delays. Automatica, 109:Article 108551, 2019.

[20] H. Lhachemi, R. Shorten, and C. Prieur. Control law realification for the feedback stabilization of a class of diagonal infinite-dimensional systems with delay boundary control. IEEE Control Systems Letters, 3(4):930-935, 2019.

[21] Z.-H. Luo, B.-Z. Guo, and O. Morgul. Stability and stabilization of infinite dimensional systems and applications. Communications and Control Engineering. Springer-Verlag, New York, 1999.

[22] A. Mironchenko and C. Prieur. Input-to-state stability of infinitedimensional systems: recent results and open questions. SIAM Review, 62(3):529-614, 2020.

[23] A. Mironchenko, C. Prieur, and F. Wirth. Local stabilization of an unstable parabolic equation via saturated controls. IEEE Transactions on Automatic Control, 2020. (To Appear) DOI:10.1109/TAC.2020.3007733.

[24] S. Misra, G. Gorain, and S. Kar. Stability of wave equation with a tip mass under unkown boundary external disturbance. Applied Mathematics E-Notes, 19:128-140, 2019.

[25] B. Perthame. Parabolic Equations in Biology: Growth, Reaction, Movement and Diffusion. Lecture Notes on Mathematical Modelling in the Life Sciences. Springer, 2015.

[26] C. Prieur and E. Trélat. Feedback stabilization of a 1D linear reactiondiffusion equation with delay boundary control. IEEE Transactions on Automatic Control, 64(4):1415-1425, 2019.

[27] F. Rothe. Global Solutions of Reaction-Diffusion Systems. Lecture Notes in Mathematics. Springer, 1984.

[28] R. Showalter. Monotone Operators in Banach Space and Nonlinear Partial Differential Equations. Mathematical Surveys and Monographs. American Mathematical Society, 1997. 